\title{
A Herd-Level Study of Risk Factors for an Elevated Prevalence of Postpartum Diseases in Dairy Farms
}

\author{
J Dubuc*, M Rousseau, J P Roy and S Buczinski \\ Université de Montréal, Faculté de médecine vétérinaire, St-Hyacinthe, Québec, Canada
}

Received: 眥 May 07, 2018; Published: 眥 May 22, 2018

*Corresponding author: Jocelyn Dubuc, Université de Montréal, Faculté de médecine vétérinaire, St-Hyacinthe, Québec, Canada

\begin{abstract}
The objective of this herd-level study was to identify risk factors for an elevated prevalence of postpartum diseases such as displaced abomasum, hyperketonemia, purulent vaginal discharge, cytological endometritis, leukocyte esterase endometritis, and prolonged anovulation. A total of 100 commercial dairy farms participated in the study for which the unit of interest was the herd. Forty cows per herd (or all of the cows calving within a 1-year period if smaller than forty cows) were enrolled in the study representing a total of 3,776 cows which were followed until they reached 60 days in milk (DIM). Cows were examined for retained placenta ( $\geq 24 \mathrm{~h}$ after calving), displaced abomasum (1-60DIM), hyperketonemia (1-14DIM), purulent vaginal discharge (30-43DIM), cytological endometritis (30-43 DIM), leukocyte esterase endometritis (30-43DIM), and prolonged anovulation (3057DIM). A number of management variables were also collected, including season, average herd size, average parity, housing systems for lactating cows and at calving, and type of bedding at calving. The prevalence of each of these diseases or management variables was computed. Logistic regression models were used to identify herd-level risk factors for the elevated prevalence of postpartum diseases in farms. Risk factors for an elevated prevalence of displaced abomasum included $\geq 5.0 \%$ retained placenta, $\geq 10.0 \%$ Hyperketonemia, smaller herd size ( $\leq 87$ lactating cows), and cold season (November to April) of calving. Risk factors for an elevated prevalence of hyperketonemia were the tie-stall housing system at calving, herd average parity $\geq 2.7$, and $\geq 5.0 \%$ retained placenta. Risk factors for an elevated prevalence of purulent vaginal discharge included $\geq 5.0 \%$ retained placenta, wood shavings as bedding at calving, and cold season (November to April) of calving. Risk factors for an elevated prevalence of cytological endometritis included $\geq 5.0 \%$ retained placenta, $\geq 10.0 \%$ hyperketonemia, and tie-stall housing system at calving. Risk factors for an elevated prevalence of leukocyte esterase endometritis were the same as for cytological endometritis. Risk factors for an elevated prevalence of prolonged anovulation were $\geq 10.0 \%$ hyperketonemia, $\geq 17.5 \%$ cytological endometritis, and cold season (November to April) of calving. Overall, an elevated prevalence of retained placenta and hyperketonemia were two common risk factors for herds with an excessive prevalence of reproductive tract diseases and prolonged anovulation.
\end{abstract}

Keywords: Dairy; anovulation; Herd-Level; Disease; Risk Factors; Postpartum; Purulent Vaginal Discharge; Leukocyte Esterase Endometritis; Cytological Endometritis; Cytobrush Technique

\section{Introduction}

Postpartum diseases are associated at the cow-level with a poorer subsequent reproductive performance. For example, postpartum metabolic disease such as hyperketonemia was shown to impair the subsequent reproductive performance of affected cows compared to unaffected animals [1-3]. Similarly, postpartum reproductive tract diseases such as cytological endometritis and purulent vaginal discharge, as well as

prolonged anovulation, were also shown to reduce the reproductive performance of affected cows [4-6]. In order to better understand and manage these diseases, many studies have investigated their risk factors at the cow-level [7-9]. Although such cow-level information is interesting to better understand the complex relationship between these conditions, it is generally more relevant for dairy farmers to manage their herds using herd-level information. In 
other words, it is known that postpartum diseases are detrimental to the subsequent performance of cows but it remains unclear at which prevalence of these diseases it is likely to negatively impact the reproductive performance of the whole herd. A recent study investigated the prevalence alarm level of postpartum diseases that was associated with poor reproductive performance at first service [10]. In summary, herds with $\geq 4.0 \%$ displaced abomasum, $\geq 11.8 \%$ hyperketonemia, $\geq 5.0 \%$ purulent vaginal discharge, $\geq 18.8 \%$ cytological endometritis, $\geq 35.3 \%$ leukocyte esterase endometritis or $\geq 21.0 \%$ prolonged anovulation were more likely to have a low first service conception risk than herds with lower prevalence of these diseases [10]. Although, these postpartum disease prevalence thresholds can be useful to explain or predict which herds are likely to have a poor reproductive performance at first service, it remains unclear for most of them what the herdlevel risk factors are for the elevated disease prevalence. Some herd-level risk factors were reported for cytological endometritis [9] and prolonged anovulation [7], but the available data generally remain scarce. Therefore, the objective of this herd-level study was to identify risk factors for the elevated prevalence of postpartum diseases that had been previously reported to be associated with a lower reproductive performance [10].

\section{Materials and Methods}

A prospective study was performed in commercial dairy herds located in the vicinity of the Faculté de médecine vétérinaire of the Université de Montréal (St-Hyacinthe, Qc, Canada). The unit of interest of this study was the herd. An estimated sample size of 98 herds was targeted based on a risk factor prevalence difference of 20 percentage points in herds with an elevated disease prevalence $(25 \%)$ compared to herds with a low disease prevalence $(5 \%)$, and accounting for alpha and beta errors of 5 and 20\%, respectively [11]. Herd selection was based on convenience, but all herds enrolled in a veterinary preventive medicine program at the bovine ambulatory clinic of the Université de Montréal (St-Hyacinthe, Qc, Canada), visited at least once a month, and using a systematic ovulation synchronization protocol for all cows at first service were contacted. The target population of this study was the population of herds enrolled in a veterinary preventive medicine program. All herds that agreed to participate in the study were visited every 14 $\mathrm{d}$ by a veterinarian and an animal health technician. In each herd, 40 cows were recruited to determine the postpartum disease prevalence. The sample size of 40 cows was based on the following assumptions: proportion expected $=6 \%, \alpha=25 \%$ (for a $75 \%$ confidence interval), and precision of estimate $=5 \%$. Within herds, enrolment of the 40 cows was systematic and performed until a total of 40 cows were recruited. In herds with a smaller size than 40 cows, all cows calving during a 1-year period were recruited. Herds were sampled between November $1^{\text {st }} 2015$ and October $31^{\text {st }} 2016$.

\section{Retained Placenta and Displaced Abomasum}

The diagnosis of retained placenta and displaced abomasum was noted for all cows. Retained placenta was diagnosed by the farmers and was defined as a retention of fetal membranes $\geq 24$ hours after calving. Farmers were trained about the definition of retained placenta prior to the start of the study on their farm. Displaced abomasum had to be diagnosed by a veterinarian within $\leq 60$ DIM and included left or right displaced abomasum cases.

\section{Hyperketonemia}

All cows were bled once between 1 and 14 DIM to collect $1 \mathrm{~mL}$ of blood from coccygeal vessels and ketonemia (BHBA) was immediately tested for using the Precison Xtra device (Abbott, Mississauga, Ontario, Canada). The analytical sensitivity of the device was $0.2 \mathrm{mmol} / \mathrm{L}$, and the maximum possible value was $7.2 \mathrm{mmol} / \mathrm{L}$. Definition of Hyperketonemia was blood BHBA $\geq 1.4 \mathrm{mmol} / \mathrm{L}[12]$.

\section{Purulent Vaginal Discharge and Cytological Endometritis}

All cows were tested for purulent vaginal discharge and cytological endometritis between 30 and 43 DIM. For vaginal discharge, the Metricheck device technique and scoring ( $0=$ no discharge, $1=$ clear mucus, $2=$ mucus with flecks of pus, $3=$ mucopurulent $(<50 \%$ pus $), 4=$ purulent discharge or $5=$ foul smelling discharge) were used [13] for endometritis, the cytobrush technique [14] was used to obtain a cytological sample from the body of the uterus. After collection, the cytobrush was rolled on a glass slide and plunged into an individual vial $(3 \mathrm{~mL})$ containing $1 \mathrm{~mL}$ of $0.9 \%$ saline solution $\mathrm{NaCl} 0.9 \%$ Irrigation, Baxter Corp. Mississauga, Ontario, Canada). A leukocyte esterase test was performed immediately on the farm using a technique described elsewhere [6]. In summary, the saline vial was shaken for $10 \mathrm{~s}$ and a drop of solution was put on a leukocyte esterase commercial strip (Multistix 10SG, Bayer Corporation, Elkart, IN). Test results were read after $2 \mathrm{~min}$ according to the manufacturer's recommendations ( 0 =negative, $0.5=$ trace amount of leukocytes, $1=$ small amount of leukocytes, $2=$ moderate amount of leukocytes, $3=$ large amount of leukocytes). The microscope glass slide of every cow that was smeared with the cytobrush was brought back to the laboratory. Within $12 \mathrm{~h}$ of collection, the slide was stained using a modified Wright-Giemsa stain (Hema3, Biochemical Sciences, Swedesboro, NJ) and a coverslip was applied when dry. Microscopic evaluation of all slides was performed individually at 400x magnification by a single experienced observer (animal health technician). The percentage of polymorphonuclear cells was determined in two different regions of the slide using a differential count of 200 cells, as previously described [6]. Cows were considered positive for purulent vaginal discharge when a Metricheck score $\geq 4$ was reported. Cows were considered positive for cytological endometritis and leukocyte esterase endometritis when $\geq 6 \%$ polymorphonuclear cells were noted on endometrial cytology and a score of $\geq 1$ for the leukocyte esterase test, respectively. 


\section{Prolonged Anovulation}

All cows were tested for prolonged anovulation by quantifying progesteronemia. Cows were bled from coccygeal vessels twice at a $14 \mathrm{~d}$ interval. The first sampling was at 30-43DIM and the second was at 44-57 DIM. Blood was collected into evacuated tubes without anticoagulant (Vacutainer, Beckton-Dixon, Franklin Lakes, NJ) that were placed on ice, brought back to the laboratory and allowed to clot. Within $6 \mathrm{~h}$ of collection, blood samples were centrifuged and serum was collected and frozen at $-20{ }^{\circ} \mathrm{C}$. Serum samples were submitted to the veterinary diagnostic service of the Université de Montréal for quantification of progesteronemia using a sequential competitive ELISA (Immulite, Siemens, Mississauga, Ontario, Canada) validated for use in cattle Martin [15] The inter- and intraassay CV of this test were 8.9 and $6.9 \%$, respectively. Cows were considered in prolonged anovulation when both samples were $<1 \mathrm{ng} / \mathrm{mL}[16]$.

\section{Management variables}

Herd-level management variables were collected, such as the season of most calving occurring during study sampling (cold season: November $1^{\text {st }}$ to April $30^{\text {th }}$; warm season: May $1^{\text {st }}$ to October $31^{\text {st }}$ ), the average number (herd size) and the average parity (herd parity) of lactating cows during the sampling period, the housing system used for lactating cows (free-stall or tie-stall), the housing system used at calving (free-stall or tie-stall), and the type of bedding used at calving (straw, wood shavings or sand).

\section{Statistical Analyses}

All statistical analyses were performed using SAS version 9.4 (SAS Institute Inc., Cary, NC). The herd was considered as the unit of interest in this study. For every herd, the prevalence of retained placenta, displaced abomasum, hyperketonemia, purulent vaginal discharge, cytological endometritis, leukocyte esterase endometritis, and prolonged anovulation were calculated. Herdlevel descriptive statistics were calculated, and values for the $25^{\text {th }}$, $50^{\text {th }}$ and $75^{\text {th }}$ percentiles were identified. A total of six outcome variables were used in the present study for which herd-level risk factors were investigated. They included an elevated prevalence of displaced abomasum $(\geq 4.0 \%)$, an elevated prevalence of hyperketonemia $(\geq 11.8 \%$ ), an elevated prevalence of purulent vaginal discharge $(\geq 5.0 \%)$, an elevated prevalence of cytological endometritis ( $\geq 18.8 \%$ ), an elevated prevalence of leukocyte esterase endometritis $(\geq 35.3 \%)$ and an elevated prevalence of prolonged anovulation $(\geq 21.0 \%)$. All of these outcomes were derived from another study investigating the prevalence of postpartum diseases associated with reproductive performance at first service and were presented elsewhere [10]. For each of these outcomes, invariable and multivariable logistic regression models were used to identify risk factors. Categorical predictors of disease prevalence were created based on the $25^{\text {th }}, 50^{\text {th }}$ and $75^{\text {th }}$ percentile values. Categorical predictors of diseases were tested in invariable models for every outcome variable based on having a biological meaning and a logical time sequence (risk factors had to occur before or at the same time as outcomes). For each outcome, only one categorical predictor of disease prevalence was selected, and selection was based on the one with the lowest P-value. Of all of the predictors selected, only those with $\mathrm{P} \leq 0.20$ were retained for further analysis. A multivariable model using the selected categorical predictors of disease prevalence was built for every outcome variable with a backward elimination strategy until all remaining variables had $\mathrm{P} \leq 0.05$. Herd management variables (season of calving, median herd size, median herd parity, housing of lactating cows, housing at calving, bedding at calving) were offered to this multivariable model as confounders and were kept as fixed effects if their impact on the model estimates was greater than $10 \%$. A total of six final multivariable models were built for which least squares means (LSM) were calculated and differences between LSM were tested using the Tukey-Kramer test. Statistical significance was defined as $\mathrm{P}<0.05$.

\section{Results}

Table 1: Descriptive statistics of herd-level prevalence from 100 herds enrolled in a study investigating risk factors for elevated prevalence of postpartum diseases in dairy herds.

\begin{tabular}{|c|c|c|c|c|c|}
\hline \multirow{2}{*}{$\begin{array}{c}\text { Herd-level } \\
\text { prevalence }\end{array}$} & Min. & Centile & Centile & Centile & \multirow{2}{*}{ Max. } \\
\cline { 3 - 5 } & $\mathbf{2 5}$ & $\mathbf{5 0}$ & $\mathbf{7 5}$ & 20.0 \\
\hline $\begin{array}{c}\text { Retained placenta } \\
\text { (\%) }\end{array}$ & 0.0 & 2.5 & 5.0 & 12.5 & 72.5 \\
\hline $\begin{array}{c}\text { Displaced } \\
\text { abomasum (\%) }\end{array}$ & 0.0 & 0.0 & 5.0 & 7.5 & 77.5 \\
\hline $\begin{array}{c}\text { Hyperketonemia } \\
\text { (\%) }\end{array}$ & 5.0 & 10.0 & 17.5 & 22.5 & 40.0 \\
\hline $\begin{array}{c}\text { Purulent vaginal } \\
\text { discharge (\%) }\end{array}$ & 0.0 & 2.5 & 5.0 & 12.5 & 77.5 \\
\hline $\begin{array}{c}\text { Cytological } \\
\text { endometritis (\%) }\end{array}$ & 5.0 & 17.5 & 30.0 & 37.5 & 80.0 \\
\hline $\begin{array}{c}\text { Leukocyte esterase } \\
\text { endometritis (\%) }\end{array}$ & 2.5 & 35.0 & 42.5 & 55.0 & 85.0 \\
\hline $\begin{array}{c}\text { Prolonged } \\
\text { anovulation (\%) }\end{array}$ & 5.0 & 22.5 & 35.0 & 52.5 & 72.5 \\
\hline
\end{tabular}

A total of 100 commercial dairy herds were enrolled in this study which represented 3,776 lactating cows. Median herd size and herd parity were 87 lactating cows (range: 31 to 371 ) and 2.7 parities (range: 1.6 to 4.3), respectively. A total of 50 herds were sampled during the cold season of calving, and the other 50 herds were sampled in the warm season of calving. Fifty-six herds had lactating cows housed in tie-stall barns whereas 44 herds were housed in free-stall barns. At calving, 67 herds had straw as bedding material whereas 30 herds had wood shavings and 3 others had sand. Also at calving, 53 herds were housed in tie-stalls, and 47 herds were housed in calving pens. Descriptive statistics (minimum, maximum, and $25^{\text {th }}, 50^{\text {th }}$ and $75^{\text {th }}$ percentiles) for the prevalence of postpartum diseases are presented in Table 1. 


\section{Elevated prevalence of displaced abomasum}

Based on previous findings, herds were stratified as having a low prevalence of displaced abomasum if $<4.0 \%(n=46)$ and as having an elevated prevalence if $\geq 4.0 \% \quad(n=54)$. Invariable logistic regression models were built to identify whether some predictors of disease prevalence were associated with it. We tested each quartile of disease predictors (centiles 25, 50 and
75 ) in these invariable models to find the one with the lowest P-value. The retained predictors of an elevated prevalence of displaced abomasum included $\geq 10.0 \%$ hyperketonemia $(\mathrm{P}<0.01)$, $\geq 5.0 \%$ retained placenta $(\mathrm{P}<0.01)$, herd size $(\mathrm{P}=0.02)$, and season of calving $(\mathrm{P}=0.03)$. We built a multivariable model from these variables and potential confounders. The final model included all of the retained predictors from invariable analyses, and the LSM from this model are presented in Figure 1.

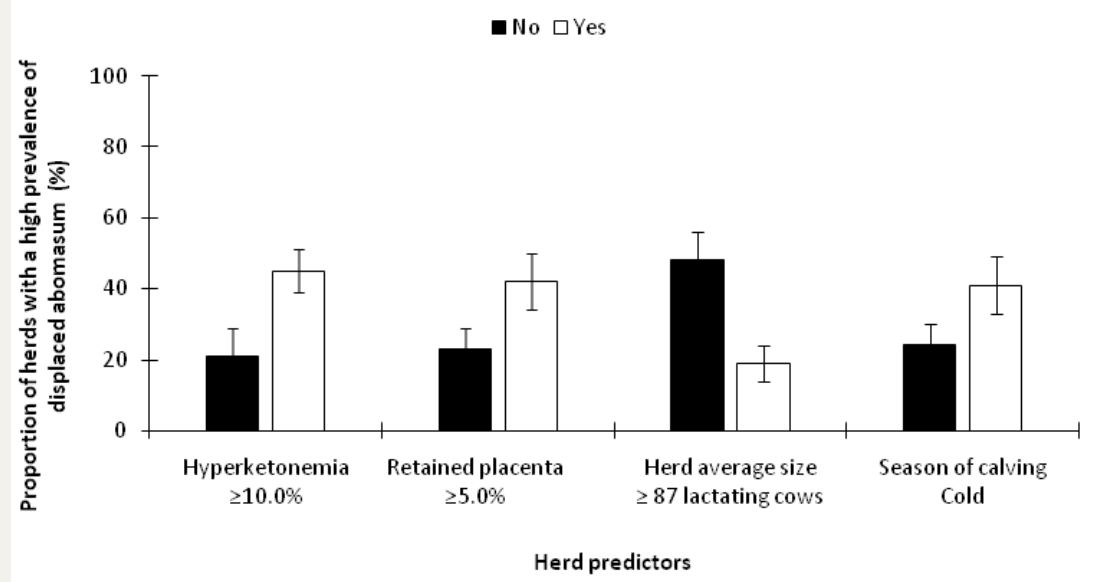

Figure 1: Least squares means ( \pm SEM) of the proportion of herds with a high prevalence of displaced abomasum in 100 dairy herds stratified by risk factors.

\section{Elevated Prevalence of hyperketonemia}

Herds were stratified as having a low prevalence of hyperketonemia if $<11.8 \%(\mathrm{n}=38)$ and as having an elevated prevalence if $\geq 11.8 \%$ ( $n=62$; [10]. The retained predictors of an elevated prevalence of Hyperketonemia included housing system used at calving $(\mathrm{P}=0.03)$, herd average parity $(\mathrm{P}<0.01)$, and $\geq 5.0 \%$ of retained placenta $(\mathrm{P}=0.01)$. The final model included all of the retained predictors from invariable analyses; the LSM from this model are presented in Figure 2.

No $\square$ Yes

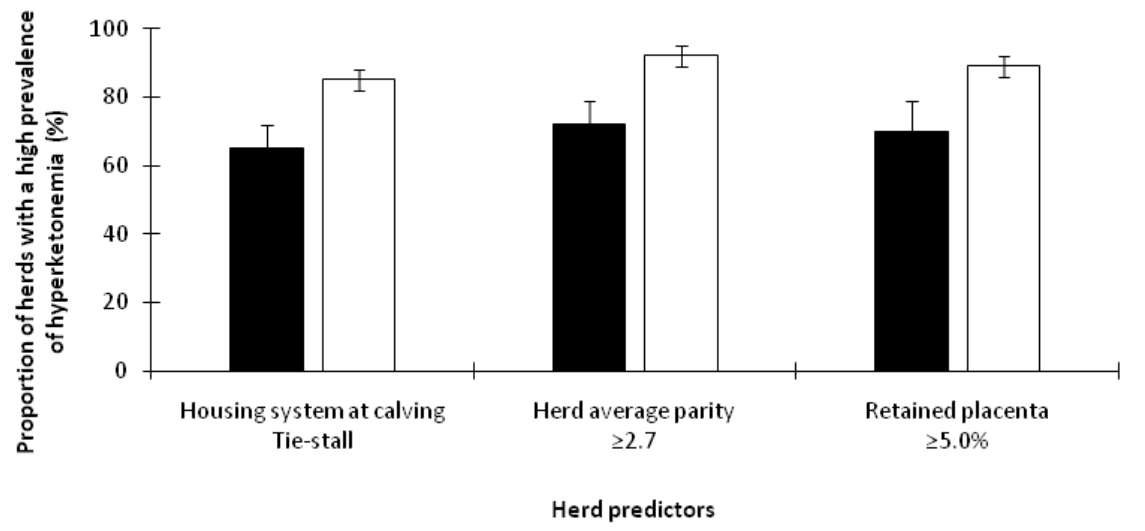

Figure 2: Least squares means $( \pm$ SEM) of the proportion of herds with a high prevalence of hyperketonemia in 100 dairy herds stratified by risk factors.

\section{Elevated Prevalence of Purulent Vaginal Discharge}

Herds were stratified as having a low prevalence of purulent vaginal discharge if $<5.0 \%(n=41)$ and an elevated prevalence if $\geq 5.0 \%(n=59 ;[10])$. The retained predictors of an elevated prevalence of purulent vaginal discharge included $\geq 5.0 \%$ retained placenta $(\mathrm{P}<0.01)$, type of bedding at calving $(\mathrm{P}=0.04)$, and season of calving $(\mathrm{P}<0.01)$. The final model included all of the retained predictors from invariable analyses; the LSM from this model are presented in Figure 3. 


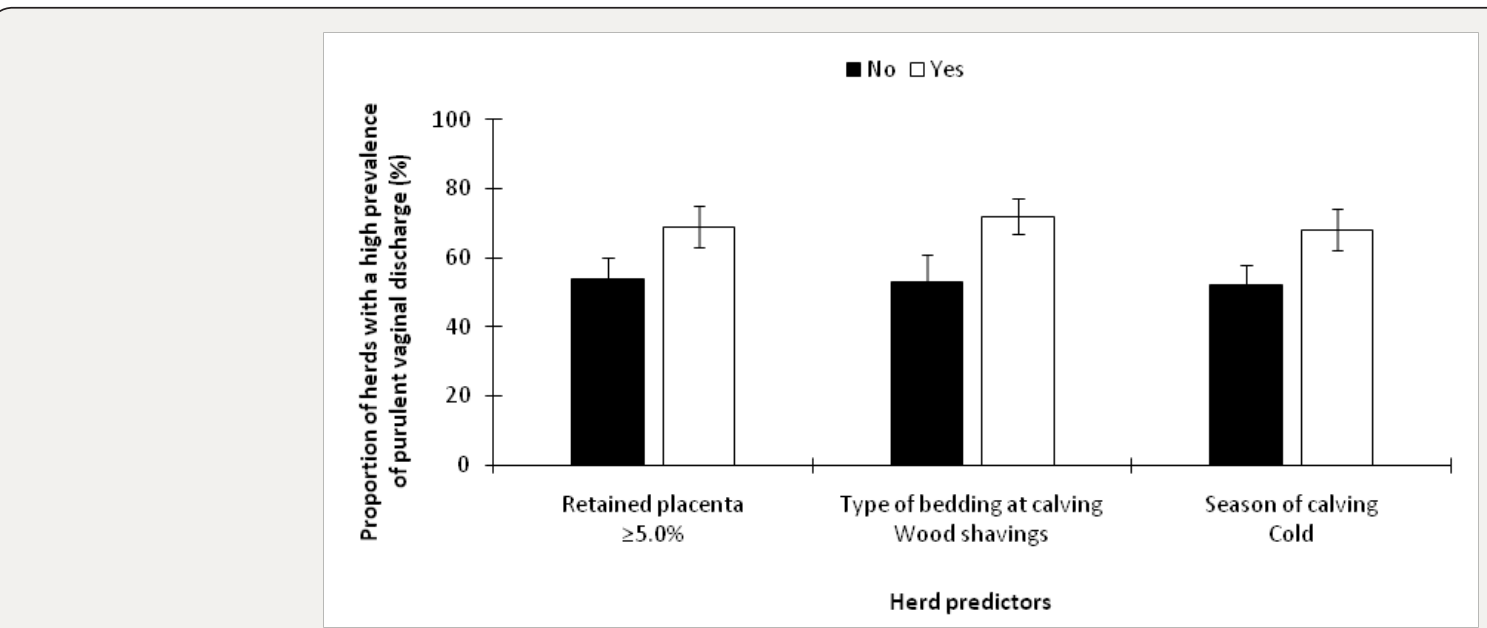

Figure 3: Least squares means ( \pm SEM) of the proportion of herds with a high prevalence of purulent vaginal discharge in 100 dairy herds stratified by risk factors.

\section{Elevated Prevalence of Cytological Endometritis}

Herds were stratified as having a low prevalence of cytological endometritis if $<18.8 \%(\mathrm{n}=29)$ and as having an elevated prevalence if $\geq 18.8 \% \mathrm{n}=71$; [10]. The retained predictors of an elevated prevalence of cytological endometritis included $\geq 5.0 \%$ retained placenta $(\mathrm{P}=0.01), \geq 10.0 \%$ hyperketonemia $(\mathrm{P}<0.01)$, and housing system at calving $(\mathrm{P}=0.02)$. The final model included all of the retained predictors from invariable analyses, and the LSM from this model are presented in Figure 4.

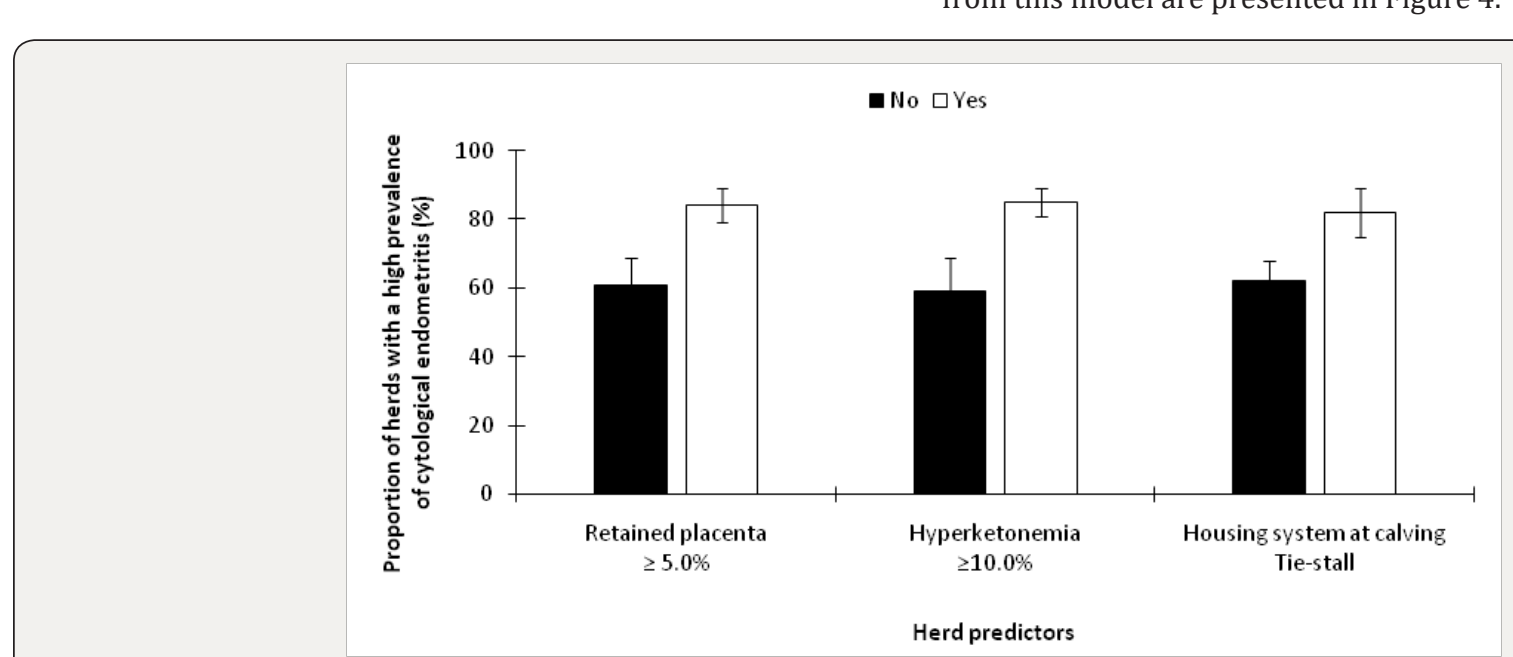

Figure 4: Least squares means $( \pm$ SEM) of the proportion of herds with a high prevalence of cytological endometritis in 100 dairy herds stratified by risk factors.

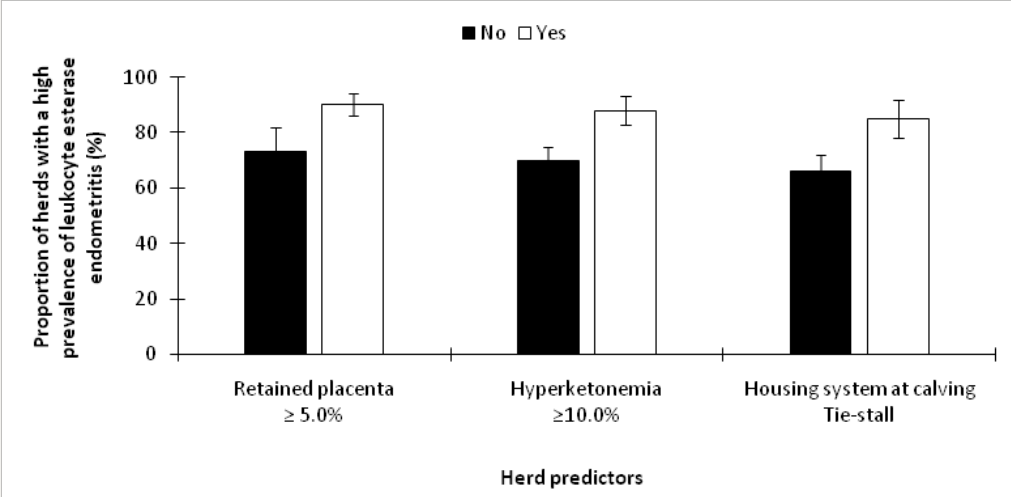

Figure 5: Least squares means ( \pm SEM) of the proportion of herds with a high prevalence of leukocyte esterase endometritis in 100 dairy herds stratified by risk factors. 


\section{Elevated Prevalence of Leukocyte Esterase Endometritis}

Herds were stratified as having a low prevalence of leukocyte esterase endometritis if $<35.3 \%(n=27)$ and an elevated prevalence if $\geq 35.3 \% n=73$; [10]. The retained predictors of an elevated prevalence of leukocyte esterase endometritis included $\geq 5.0 \%$ retained placenta $(\mathrm{P}<0.01), \geq 10.0 \%$ hyperketonemia $(\mathrm{P}=0.02)$, and housing system at calving $(\mathrm{P}=0.04)$. The final model included all of the retained predictors from invariable analyses; the LSM from this model are presented in Figure 5.

\section{Elevated Prevalence of Prolonged Anovulation}

Herds were stratified as having a low prevalence of prolonged anovulation if $<21.0 \%(n=21)$ and an elevated prevalence if $\geq 21.0 \%$ $(n=79 ;[10])$. The retained predictors of an elevated prevalence of prolonged anovulation included $\geq 10.0 \%$ hyperketonemia ( $\mathrm{P}=0.02$ ), $\geq 17.5 \%$ cytological endometritis $(\mathrm{P}<0.01), \geq 35.0 \%$ of leukocyte esterase endometritis $(\mathrm{P}<0.01)$, and season of calving $(\mathrm{P}<0.01)$. The final model included all of the retained predictors from invariable analyses except leukocyte esterase endometritis; the LSM from this model are presented in Figure 6.

No $\square$ Yes
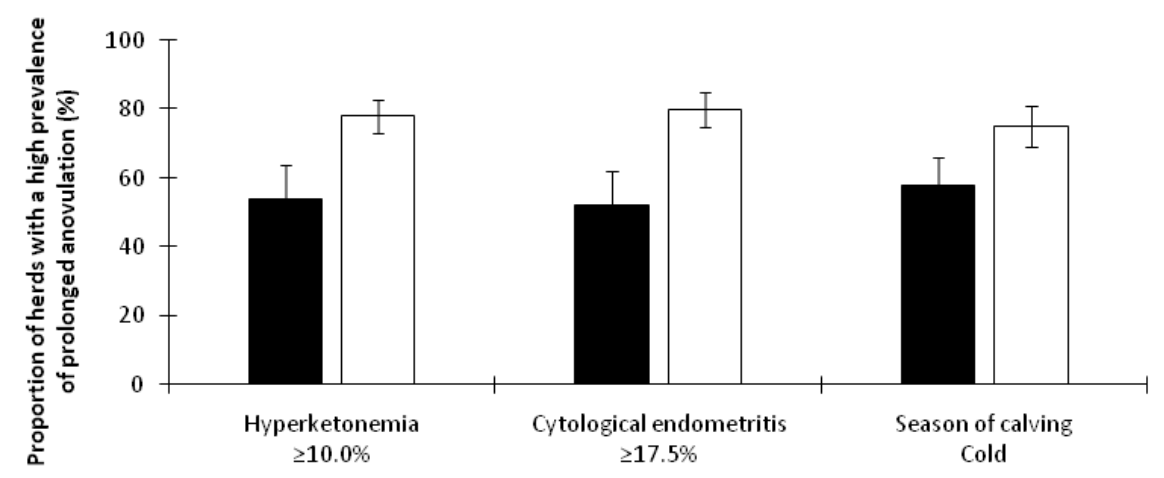

Herd predictors

Figure 6: Least squares means $( \pm$ SEM) of the proportion of herds with a high prevalence of prolonged anovulation in 100 dairy herds stratified by risk factors.

\section{Discussion}

The body of literature describing herd-level risk factors for the elevated prevalence of postpartum diseases remains small, even if this information can be extremely useful for dairy farmers and their advisors for decision-making. Some important bottlenecks that could explain this situation are the difficulty to recruit a large number of herds and to capture herd-level information that is not routinely collected by farmers (e.g. feed intake, fed-ration composition, etc.). Using such a large number of herds implies that these studies have to be performed on commercial farms and not in research stations where almost all desirable data can be collected. Although the current study was designed prospectively, it needs to be kept in mind that the capture of much of the ideal information was not possible because of the inaccuracy or unavailability of these data. However, future studies should consider the use of herd-level study design more often because associations found at the cow-level do not automatically hold true at the herd-level. Not considering it is known as an atomistic fallacy and should be avoided. Although not perfect, the current study was designed to identify herd-level risk factors for herds with an elevated prevalence of some postpartum diseases. Interestingly, it was possible to identify risk factors for all study outcomes (diseases) that were targeted. Risk factors for an elevated prevalence of displaced abomasum included $\geq 5.0 \%$ retained placenta, $\geq 10.0 \%$ hyperketonemia, small $(<87$ ) herd size, and cold season of calving. The association between displaced abomasum and Hyperketonemia has already been shown in cowand herd-level studies [17-19]. One could speculate that cows in excessive negative energy balance are more likely to develop displaced abomasum because they eat less, which may leave more room for the abomasum to move in the abdomen of cows. The relationship between retained placenta and displaced abomasum was described at the cow-level [20], but had not been described at the herd-level. It seems unlikely that a retained placenta by itself would cause displaced abomasum, but it might be associated with displaced abomasum because of the relationship with energy balance and dry matter intake.

The finding that the prevalence of displaced abomasum is generally higher in smaller herds is interesting. Perhaps the peripartum nutritional strategy is more difficult to optimize in smaller herds than in larger ones, as it is generally hard to have a specific and consistent ration during the peripartum period when only a small number of cows are fed with that ration. Regarding the season effect, it may seem at first counterintuitive that the warm season of calving, which includes a period of heat stress and its impact on dry matter intake, is not associated with a higher prevalence of displaced abomasum when compared with the cold 
season. However, it should be kept in mind that the magnitude of heat stress in the summer months in the province of Québec (Canada) is much smaller than if the study had been conducted in the southern USA or other hotter locations in the world. In the northern country situation of Canada, it could be speculated that the peripartum transition period might be more negatively influenced by the very cold weather conditions during winter than by the heat stress period that is generally relatively short. Further research should explore this finding.

Risk factors for an elevated prevalence of hyperketonemia found in the present study included $\geq 5.0 \%$ retained placenta, tie-stall housing systems at calving, and high $(\geq 2.7)$ herd average parity. As previously described, the association between hyperketonemia and retained placenta is not surprising, but might be better understood in future studies if the actual dry matter intact information can be captured, as it is likely to be one of the main causes of this association. A high herd average parity generally implies that the proportion of older cows is large and it is known that older cows are more likely to experience hyperketonemia Chapinal [18]. Therefore, it seems biologically plausible that herd average parity is associated with hyperketonemia. It remains unclear why the tie-stall housing system would be associated with the greater prevalence of hyperketonemia but it could be speculated that the feeding program and strategy might be different. Further research is needed in this field. Risk factors found for an elevated prevalence of reproductive tract disease in herds are interesting. As expected, a high $(\geq 10.0 \%)$ prevalence of Hyperketonemia was retained in the final models for cytological endometritis and leukocyte esterase endometritis but not in the one for purulent vaginal discharge. It had already been highlighted in cow-level studies that hyperketonemia was associated with cytological endometritis $[8,9]$ but not with purulent vaginal discharge [8]. Also, as expected, $\geq 5.0 \%$ prevalence of retained placenta was found in all final models. This relationship could be explained by the increase in reproductive tract bacterial contamination and purulent material found in the reproductive tract during the postpartum period. The use of wood shavings was found to be a risk factor for herds with an elevated prevalence of purulent vaginal discharge. Perhaps the use of wood shaving increases the magnitude of reproductive tract bacterial contamination during the early postpartum period, which subsequently increases the risk of purulent vaginal discharge. This finding deserves further investigation. Another interesting finding was that the risk factors for cytological endometritis and leukocyte esterase endometritis were the same. Therefore, the hypothesis raised in a previous study [10], which states that although these two tests do not provide the exact same information at the cow level elevated herd-level prevalence of these diseases might the result of similar causes, is confirmed. It reinforces the usefulness of the surveillance of leukocyte esterase endometritis in dairy farms when monitoring of cytological endometritis is not possible. Accounting for the effect of season, risk factors for an elevated prevalence of prolonged anovulation in herds included $\geq 10.0 \%$ hyperketonemia, $\geq 17.5 \%$ cytological endometritis and $\geq 35.0 \%$ leukocyte esterase endometritis in invariable models. The final model only included the variables hyperketonemia and cytological endometritis because there was colinearity between the latter and leukocyte esterase endometritis. The association between prolonged anovulation and negative energy balance as well as reproductive tract disease has been reported in cow-level studies $[7,21,22]$ and is not surprising. The results from this project should be useful for helping to identify potential risk factors when the prevalence of postpartum disease in herds is too high. From a farm management perspective, it might be more useful to identify herds that are at high risk of subsequently elevated disease prevalence as early as possible. For example, one could perform a systematic surveillance of the prevalence of retained placenta and hyperketonemia in herds to identify which ones are at greater risk of having an elevated prevalence of reproductive tract disease and prolonged anovulation. Combined with other herdlevel study results, this information should help to aim for optimal performance in milk production, health, reproduction and survival in dairy herds.

\section{Conclusion}

Herd-level risk factors for many postpartum diseases were identified in a population of 100 commercial dairy farms. An elevated prevalence of retained placenta or hyperketonemia was common risk factors for herds with an elevated prevalence of reproductive tract disease and prolonged anovulation. Future research should consider performing a similar herd-level study including peripartum calcium and non-esterified fatty acid measurements to provide a more complete picture of their impacts.

\section{References}

1. Walsh RB, JS Walton, DF Kelton, SJ LeBlanc, KE Leslie, et al.(2007) The effect of subclinical ketosis in early lactation on reproductive performance of postpartum dairy cows. J Dairy Sci 90(6): 2788-2796.

2. Ospina PA, DV Nydam, T Stokol, TR Overton (2010) Associations of elevated non-esterified fatty acids and $\beta^{2}$-hydroxybutyrate concentrations with early lactation reproductive performance and milk production in transition dairy cattle in the northeastern United States. J Dairy Sci 93(4): 1596-1603.

3. McArt JAA, D. V. Nydam, GR Oetzel (2012) Epidemiology of subclinical ketosis in early lactation dairy cattle. J Dairy Sci 95(9): 5056-5066.

4. Dubuc J, TF Duffield, KE Leslie, JS Walton, SJ LeBlanc (2010) Definitions and diagnosis of postpartum endometritis in dairy cows. J Dairy Sci 93(11): 5225-5233.

5. Vieira-Neto A, RO Gilbert, WR Butler, JEP Santos, ES Ribeiro, et al.(2014) Individual and combined effects of anovulation and cytological endometritis on the reproductive performance of dairy cows. J Dairy Sci 97(9): 5415-5425.

6. Denis-Robichaud J, J Dubuc (2015) Determination of optimal diagnostic criteria for purulent vaginal discharge and cytological endometritis in dairy cows. J Dairy Sci 98(10): 6848-6855.

7. Walsh RB, DF Kelton, TF Duffield, KE Leslie, JS Walton, et al.(2007) Prevalence and risk factors for postpartum anovulatory condition in dairy cows. J Dairy Sci 90(1): 315-324. 
8. Dubuc J, TF Duffield, KE Leslie, JS Walton, SJ LeBlanc (2010) Risk factors for postpartum uterine diseases in dairy cows. J Dairy Sci 93(12): 5764 5771

9. Cheong SH, DV Nydam, KN Galvão, BM Crosier, RO Gilbert (2011) Cowlevel and herd-level risk factors for subclinical endometritis in lactating Holstein cows. J Dairy Sci 94(2): 762-770.

10. Dubuc J, J Denis-Robichaud (2017) A dairy herd-level study of postpartum diseases and their association with reproductive performance and culling. J Dairy Sci 100(4):3068-3078.

11. Dohoo I, W Martin, H Stryhn (2003) Veterinary Epidemiologic Research. $1^{\text {st }}$ Edition. AVC Inc., Charlottetown, Canada, PE.

12. Duffield TF, KD Lissemore, BW McBride, KE Leslie (2009) Impact of hyperketonemia in early lactation dairy cows on health and production. J Dairy Sci 92(2):571-580.

13. McDougall S, R Macaulay, C Compton (2007) Association between endometritis diagnosis using a novel intravaginal device and reproductive performance in dairy cattle. Anim Reprod Sci 99(1-2): 9-23.

14. Kasimanickam R, TF Duffield, RA Foster, CJ Gartley, KE Leslie, et al. (2004) Endometrial cytology and ultrasonography for the detection of subclinical endometritis in postpartum dairy cows. Theriogenology 62(1-2): 9-23.

15. Martin JL, KA Vonnahme, DC Adams, GP Lardy, RN Funston (2007) Effects of dam nutrition on growth and reproductive performance of heifer calves. J Anim Sci 85(3): 841-847.
16. Stevenson JS, JR Pursley, HA Garverick, PM Fricke, DJ Kesler, et al. (2006) Treatment of cycling and non-cycling lactating dairy cows with progesterone during ovsynch. J Dairy Sci 89(7): 2567-2578.

17. Ospina PA, DV Nydam, T Stokol, TR Overton (2010) Association between the proportion of sampled transition cows with increased non-esterified fatty acids and $\beta$-hydroxybutyrate and disease incidence, pregnancy rate, and milk production at the herd level. J Dairy Sci 93(8): 3595-3601.

18. Chapinal N, M Carson, TF Duffield, M Capel, S Godden, et al. (2011) The association of serum metabolites with clinical disease during the transition period. J Dairy Sci 94(10): 4897-4903.

19. Chapinal N, SJ LeBlanc, ME Carson, KE Leslie, S Godden, et al. (2012) Herd-level association of serum metabolites in the transition period with disease, milk production, and early lactation reproductive performance. J Dairy Sci 95(10): 5676-5682.

20. Correa MT, H Erb, J Scarlett (1993) Path analysis for seven postpartum disorders of Holstein cows. J Dairy Sci 76(5): 1305-1312.

21. Sheldon IM, J Cronin, L Goetze, G Donofrio, HJ Schuberth (2009) Defining Postpartum Uterine Disease and the Mechanisms of Infection and Immunity in the Female Reproductive Tract in Cattle. Biol Reprod 81(6): 1025-1032.

22. Dubuc J, TF Duffield, KE Leslie, JS Walton, SJ LeBlanc (2012) Risk factors and effects of postpartum anovulation in dairy cows. J Dairy Sci. 95(4): 1845-1854.

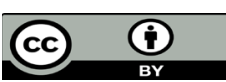

This work is licensed under Creative

Commons Attribution 4.0 License

Submission Link:

$$
\text { Submit Article }
$$

DOI: $10.32474 / C D V S .2018 .01 .000116$

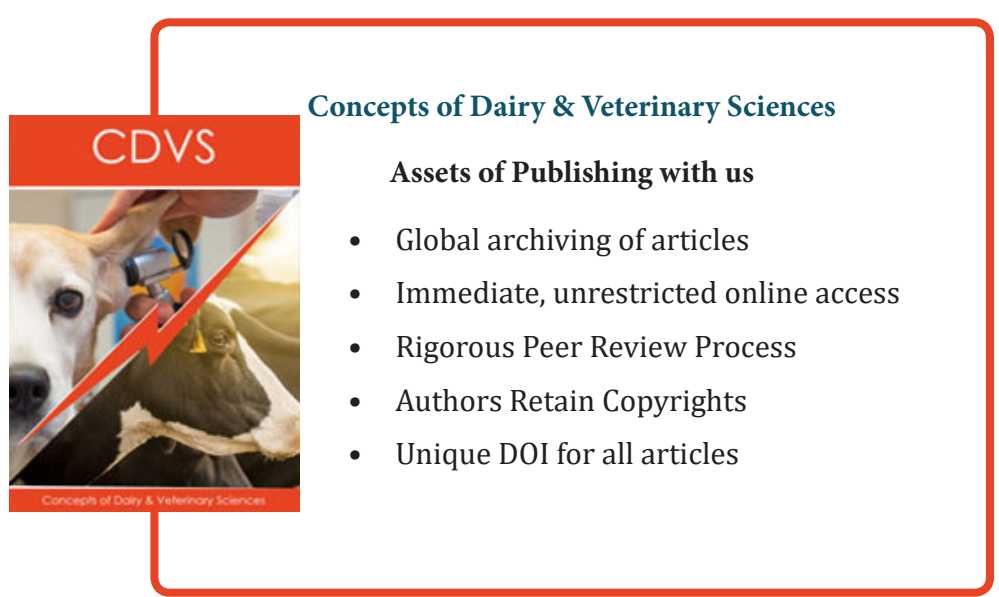

